\title{
AN APPROACH FOR MULTISPECTRAL IMAGE COMPRESSION
}

\author{
Dr.H S Prashantha \\ Professor, E\&CE department \\ Nitte Meenakshi Institute of Technology \\ Bangalore-64, Karnataka, India
}

\begin{abstract}
Compression plays a key role in reducing transmission and storage requirements. Compression of Multispectral data is important as it demands lots of memory space for storage. In the present work, the paper proposes the use of two transform coding techniques such as Discrete Wavelet Transform (DWT) and Discrete Cosine Transform (DCT) for compressing the multispectral data. The two transform coding techniques are applied for multispectral input data and are compared with respect to Compression Ratio (CR) and Peak Signal to Noise Ratio (PSNR). Both the techniques are implemented individually on various bands of multispectral image.
\end{abstract}

Keywords- Multispectral image, Discrete cosine transform, Discrete wavelet transform, Compression

\section{INTRODUCTION}

Image compression is a type of data compression applied to digital images, to reduce their cost for storage or transmission. Its aim is to reduce the number of bits required to represent an image by removing the spatial and spectral components to lowest possible level. The redundancy and irrelevancy that is present in the image will be reduced during image compression. It aims in reducing the size of multispectral image using image compression techniques. The objective is to reduce the transmission and storage requirements of image data. The two types of compression which describes image compression are:

Lossless compression: This type of compression reduces the size of the image by changing the way in which the data is stored. Therefore this type of compression will make no changes in your image. They do not add noise to the image.

Lossy compression: In this type of compression, the size of the image will be reduced by removing some data from it which in turn reduces the quality of the image but will not affect the quality of that image.

A multispectral image is one that captures image data within specific wavelength ranges across the electromagnetic spectrum. The wavelengths may be separated by filters or detected via the use of instruments that are sensitive to particular wavelengths, including light from frequencies

\author{
Nagesh K N, Karthik H, Ankith Shetty R, Likitha C \\ Undegraduate Student, E\&CE department \\ Nitte Meenakshi Institute of Technology \\ Bangalore-64, Karnataka, India
}

beyond the visible light range, i.e., infrared and ultra violet. Extraction of additional information is allowed in spectral imaging when the human eye fails to capture red green and blue with its visible receptors. A single image band in multispectral image may occupy hundreds of megabytes for storage and to transmit these images becomes huge. To overcome these problems, multispectral image compression is required. The multispectral image is decomposed into individual spectral bands and compression techniques like DCT, DWT is used to compress these spectral bands.

The contribution made by different authors are presented to understand the research gap. A.M.Raid, W.M.Khedr, M. A. El-dosuky and Wesam Ahmed presented a survey paper on lossy image compression using Discrete Cosine Transform, quantization, entropy encoding. Jin Li, Fei Xing, Ting Sun, and Zheng You presented a research paper in which a compression algorithm based on distributed source coding (DSC) combined with image data compression (IDC) approach recommended by CCSDS for multispectral images. Arto Kaarna and Jussi Parkkinen presented three methods for the lossy compression of multispectral images are developed. It compares the properties of those methods by applying them to several multispectral images. Bhagya Raju, Dr. K. Jaya Sankar, Dr. C. D. Naidu and Srinivas Bachu presented a paper on improved SPIHT algorithm and fuzzy based SR image reconstruction. The method incorporated a new way to overcome the limitation that was prevalent with compression and transmission. The fuzzy based approach is more effective and proves to be likely applicable for multi spectral images. S. Boopathiraja, P. Kalavathi presents a near lossless compression method used for multispectral images. ThreeDimensional Discrete Wavelet Transform is used for decomposition and the Huffman coding followed by thresholding is used for encoding. The results of our proposed method for the multispectral LANDSAT images are discussed and compared with other existing methods in terms of PSNR and SSIM. R. Kavin Rajesh, C.Heltin Genitha discussed about compression techniques using DWT, SPIHT and Huffman coding and reconstruction of image by using Inverse operations. M. Klimesh presented two algorithms KarhunenLoeve transform (KLT) in the spectral dimension, the discrete cosine transform (DCT) for spatial decorrelation of the 
resulting bands, and the DCT on the residual. H S Prasanrha and others discussed about Image compression using SVD for image compression. M J Raghavendra and others discussed about image compression using hybrid combinations of DCT and RLE.

The rest of the paper is organized as follows. Proposed Methodology are explained in section II. Experimental results are presented in section III. Concluding remarks are given in section IV.

\section{PROPOSED ALGORITHM}

\section{A. Discrete Cosine Transform}

Discrete cosine transform (DCT) is one of the most popular transforms in image processing. It is widely used for image compression because of its high energy packing capabilities. The spatial domain image is converted into frequency domain image using DCT. Image is represented as a sum of sinusoidal of varying magnitudes and frequencies. At high data compression ratio, DCT is capable of achieving higher quality. This property is termed as energy compaction. Lossy image compression uses discrete cosine transform for image compression.

For compression each spectral band is divided into $8 \times 8$ blocks and DCT is applied. After masking most of the values in the block becomes zero. Masking is done to remove the high frequency component in order to reduce the pixels and to save memory. For reconstruction unmasking and inverse DCT is applied.

\section{B. Discrete Wavelet Transform}

A discrete time signal is transformed into a discrete wavelet representation using Discrete Wavelet Transform (DWT) and are used for wavelet-based compression and coding. Decomposition level indicates a band of frequency. So if you increase the number of decomposition level then each band will be narrower which means you will have better frequency resolution. Hence the decomposition level can either be increased or decreased as per the requirement. In spatial frequency domain, the wavelet filter allows to emphasize or de-emphasize the image details. Thresholding is a simple, yet effective, way of partitioning an image. It is applied to remove the pixel of the image to compress the image. After thresholding the compressed image is obtained. Same steps is applied for all the spectral bands. Inverse discrete wavelet transform (IDWT) is applied at the receiver to get back the original image.

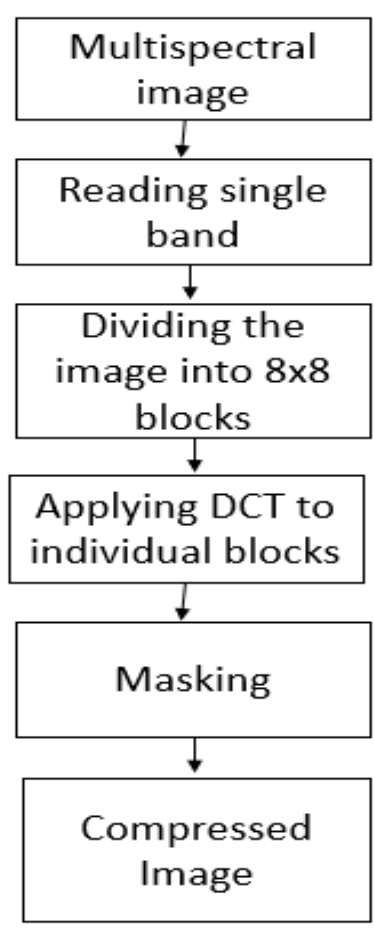

Fig. 1 Multispectral Image Compression using DCT

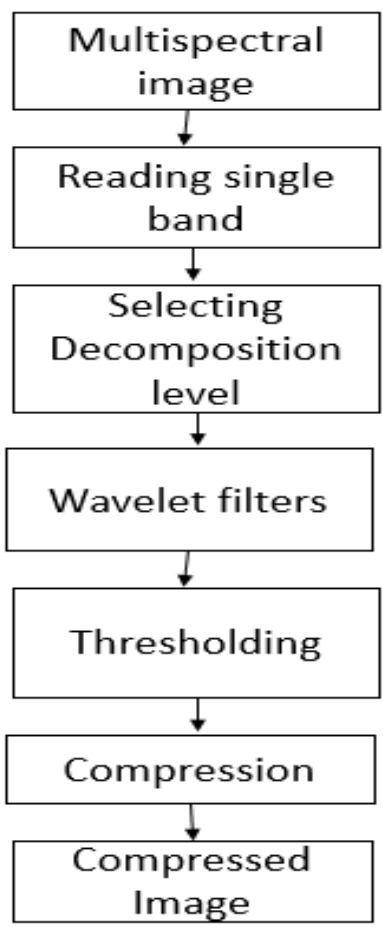

Fig. 2 Multispectral Image Compression using DWT 


\section{EXPERIMENT AND RESULT}

The test set for this evaluation experiment is performed on MATLAB 7.0 software platform and the PC for experiment is equipped with an Intel P4 2.4 GHz Personal laptop and 2GB memory. The proposed scheme is tested using multispectral images for both DCT and DWT Techniques.

\section{RESULTS OF DWT (Different Wavelets)}

DWT using different wavelets are applied on individual bands of multispectral image and compressed images and reconstructed images are obtained. Performance parameters like compression ratio and PSNR are also calculated for each band. The experiments are conducted for all the 8-bands and the sample result for band- 1 is displayed for analysis.

\section{For BAND - 1}
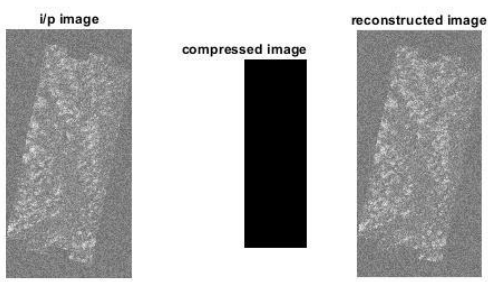

Figure 3. Input, compressed and reconstructed image of wavelet - Daubechies (db1) and its decomposition level-2
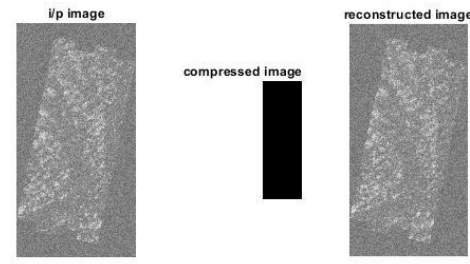

Figure 4. Input, compressed and reconstructed image of wavelet - Daubechies (db1) and its decomposition level- 3
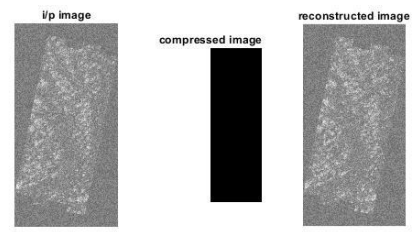

Figure 5. Input, compressed and reconstructed image of wavelet - discrete meyer and its decomposition level - 2

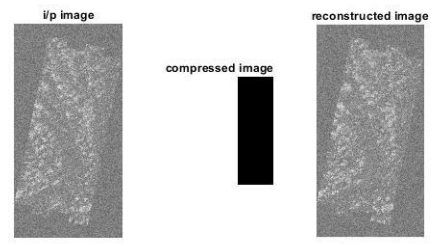

Figure 6. Input, compressed and reconstructed image of wavelet - discrete meyer and its decomposition level - 3

Table 1: Comparison of CR and PSNR for different wavelets

\begin{tabular}{|c|c|c|}
\hline Figure & Compression ratio & PSNR \\
\hline 3 & 83.33 & 25.2022 \\
\hline 4 & 75 & 25.2029 \\
\hline 5 & 50 & 25.2025 \\
\hline 6 & 33.33 & 25.2021 \\
\hline
\end{tabular}

The table 1 shows the comparison result in terms of compression ratio and PSNR for band 1

\section{RESULTS OF DCT}
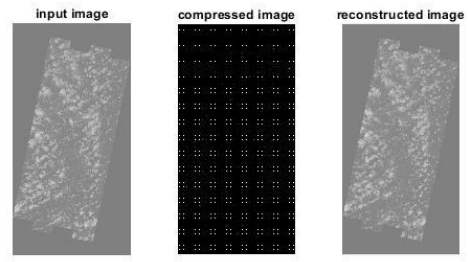

Figure 7: Input, compressed \& reconstructed image of band-1
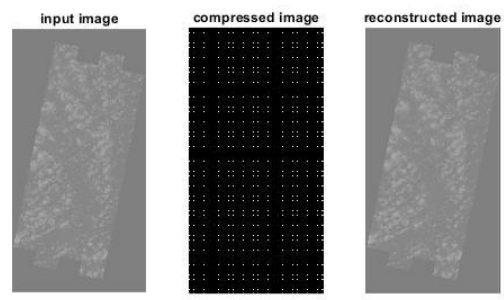
Figure 8: Input, compressed \& reconstructed image of band 2
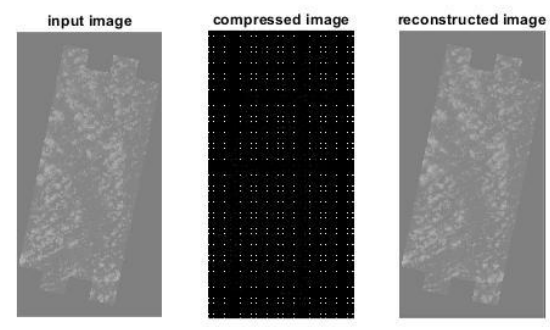

Figure 9: Input, compressed \& reconstructed image of band 3

Table 2: Comparison of CR and PSNR for different bands

\begin{tabular}{|c|c|c|}
\hline Figure & Compression ratio & PSNR \\
\hline 7 & 87.4908 & 34.1299 \\
\hline 8 & 87.4783 & 35.5121 \\
\hline 9 & 87.4783 & 34.8367 \\
\hline
\end{tabular}

For low compression ratio Discrete Cosine Transform should be used because implementation of DCT is less expensive than that of the DWT. For higher compression ratio DCT cannot be used because of very poor image Quality as it introduces blocking artifacts.

\section{CONCLUSION}

Multispectral image compression is implemented using both Discrete Wavelet Transform and Discrete Cosine Transform techniques and simulated using MATLAB software. Multispectral image is separated into various spectral bands and then the algorithm is applied. Both the techniques are applied for all bands of the multispectral image and reconstruction is obtained and the results are tabulated. Compression ratio and PSNR are also obtained for each band for better understanding.

Further it is possible to obtain compression for the entire multispectral image instead of individual bands. Also other techniques such as SVD, Hadamard transform, etc. can be tried for compression and obtain comparative study of the compression techniques.

\section{REFERENCE}

1. Gunasheela K S, H S Prasantha, "Satellite image compressiondetailed survey of the algorithms",
Proceedings of ICCR in LNNS Springer, 2017, vol. 14, pp. 187-198.

2. V. Bhagya raju, K.Jaya sankar, C.D.Naidu,srinivas bachu ,"Multispectral image compression with high resolution improved spiht for testing various input images," January - March 2016

3. C.Senthilkumar, S.Pannirselvam ", Image Compression for Color and Multispectral Image using Enhanced BTC "International Journal of Computer Applications (0975 - 8887) National Conference on Research Issues in Image Analysis \& Mining Intelligence (NCRIIAMI-2015)

4. Tushar gupta, A Nithyakalyani, Utkarsh Rahim, "Analysus and improvement of digital image compression using DCT algorithm" Intenational Journal of pure and applied mathematics 2018

5. Friedhelm König, Agfa-Geveart AG, Munich, Germany, "Compression of Multispectral Images Combining Spatial and Spectral Compression". IS\&T's 2003.

6. Friedhelm König and Werner Praefcke, Multispectral image encoding, Proc. of IEEE's ICIP'99 International Conference on Image Processing, volume 3. pp. 45-49, Kobe, Japan, 1999.

7. H. S. Prasantha, H. L. Shashidhara, and K. N. Balasubramanya Murthy. Image compression using SVD. In Proceedings of the International Conference on Computational Intelligence and Multimedia Applications, pages 143-145. IEEE Computer Society, 2007.

8. Raghavendra.M.J, Prasantha.H.S and S.Sandya, "Image Compression Using Hybrid Combinations of DCT SVD and RLE", International Journal of Computer Techniques, Volume 2 Issue 5-2015.

9. Gunasheela K S, H S Prasantha, "Compressive sensing for image compression: survey of algorithms", Proceedings of Emerging Research in Computing, Information, Communication and Applications, ERCICA, Springer publication, Bengaluru, 2018

10. K N Shruthi, B M Shashank, Y. SaiKrishna Saketh, H.S Prasantha and S. Sandya, "Comparison Analysis Of A Biomedical Image For Compression Using Various Transform Coding Techniques", IEEE, pp. 297-303, 2016

11. Image Data Compression. Issue 2. Report Concerning Space Data System Standards

12. (Green Book), CCSDS 120.1-G-2. Washington, D.C.: CCSDS, February 2015.

13. S.G. Mallat, "A Theory for Multiresolution Signal Decomposition: The Wavelet Representation," IEEE Trans. Pattern Analysis and Machine Intelligence, vol. 11, no. 7, pp. 674-693, July 1989. 